\title{
DISLOCATION NUCLEATION AT METAL-CERAMIC INTERFACES
}

\author{
G. E. BELTZ† and J. R. RICE \\ Division of Applied Sciences, Harvard University, Cambridge, MA 02138, U.S.A.
}

\begin{abstract}
The ductile vs brittle behaviour of metal-ceramic interfaces is discussed within an atomistic framework, in which the mechanical response of an interfacial crack is assumed to be ultimately controlled by the competition between atomic decohesion and dislocation nucleation ahead of the crack tip. As in later versions of the Rice-Thomson model, this competition may be evaluated in terms of the parameters $G_{\text {cleave }}$, the energy release rate for cleavage of the metal-ceramic interface, and $G_{\text {diss }}$, the energy release rate associated with the emission of a single dislocation within the metal. The various models of dislocation nucleation are discussed, with emphasis on an approach which makes use of Peierls-like stress vs displacement relations on a slip plane ahead of a crack tip. A recent analytical result by Rice shows that for a mode II or III shear crack, with a slip plane parallel to the crack plane, a dislocation is emitted when $G=\gamma_{u s}$ ( $G$ is the energy release rate corresponding to the "screened" crack tip stress field and $\gamma_{u s}$ is the "unstable stacking" energy asssociated with the sliding of atomic planes past one another). This treatment permits the existence of an extended dislocation core, which eliminates the need for the core cutoff radii required by the Rice-Thomson model of emission. Results are presented here for the nucleation of dislocations under more realistic assumptions for metal-ceramic cracks, namely, the emission on inclined slip planes within a mixed-mode crack-tip field. The specific case of a copper crystal bonded on a $\{221\}$ face to sapphire is analyzed, and the results are used to interpret the recent experimental observations of Beltz and Wang [Acta metall. mater. 40, 1675 (1992)] on directional toughness along this type of interface.
\end{abstract}

Résumé-Le comportement fragile-ductile des interfaces métal/céramique est discuté en supposant que la réponse mécanique d'une fissure interfaciale est contrôlée finalement par la compétition entre la décohésion atomique et la germinaton de dislocations en avant de l'extrêmité de la fissure. Comme dans les denières versions due modèle de Rice et Thomson, cette compétition peut être évaluée en fonction du paramètre $G_{\text {cliv. }}$, du taux de libération de l'énergie pour le clivage de l'interface métal/céramique et de $G_{\text {disl }}$, le taux de libération de l'énergie associée à l'émission d'une seule dislocation à l'intérieur du métal. Les différents modèles de germination de dislocations sont discutés, en insistant sur une approche qui utilise une pseudo contrainte de Peierls, fonction des relations de déplacement dans un plan de glissement en avant de l'extrêmité d'une fissure. Un résultat analytique récent de Rice montre que pour un mode II ou III de fissure de cisaillement, avec un plan de glissement parallèle au plan de la fissure, une dislocation est émise lorsque $G=\gamma_{\mathrm{ei}}$ ( $G$ est le taux de libération de l'énergie correspondant au champ de contrainte "écranté" de l'extrêmité de la fissure et $\gamma_{u s}$ est l'énergie d"'empilement instable" associée au glissement des plans atomiques l'un sur l'autre). Ce traitement permet l'existence d'un coeur de dislocation étendu, ce qui élimine la nécessité de connaitre les rayons de coupure du coeur exigés par le modèle de Rice et Thomson. Les résultats sont présentés ici pour la germination de dislocations avec des hypothèses pus réalistes pour des fissures métal/céramique, c'est-à-dire l'émission sur des plans de glissement inclinés dans un champ de mode mixte à l'extrêmité de la fissure. Le cas spécifique d'un cristal de cuivre lié sur une face $\{221\}$ à du saphir est analysé et les résultats sont utilisés pour interprêter les observations expérimentales récentes de Beltz et Wang [Acta metall. mater. 40, 1675 (1992)] sur la ténacité directionnelle sur ce type d'interface.

Zusammenfassung-Das duktile Bruchverhalten im Gegensatz zum spröden von Grenzflächen zwischen metallischen und keramischen Werkstoffen wird im atomistischen Rahmen besprochen unter dem Gesichtspunkt, daß der mechanische Reaktions-ablauf eines Grenzfiächenrisses letztlich von der Konkurrenz zwischen atomistischen Spaltungsbruch und Versetzungskeimbildung vor der Rißspitze abhängt. Wie in späteren Versionen des Rice-Thomson Modells, könnte diese Konkurrenz bewertet werden nach Parametern $G_{\text {ckave }}$, die Energiefreigaberate für Spaltungsbruch der metall-keramischen Grenzfläche, und $G_{\text {diel }}$, die Energiefreigaberate für Emission im Metall einer einzelnen Versetzung. Es werden die verschiedenen Modelle von Versetzungskeimbildung besprochen, mit Betonung auf eine Methode in der ein Peirlsähnlicher Spannung-Verschiebungszusammenhang auf einer Gleitebene vor einer Rißspitze verwendet wird. Ein vor kurzem analytisches Ergebniss von Rice zeigt, daß für Mode II order III Schubrisse mit Gleitebene parallel mit der Rißebene, wenn $G=\gamma_{u s}$ besteht ( $G$ ist die Energiefreigaberate in Bezug auf das locale plastisch "abgeschirmte" Rißspitzenspannungsfeld und $\gamma_{u s}$ ist die "unbeständige Stapelenergie" die mit dem Aneinandervorbeigleiten von atomistischen Ebenen verbunden ist), eine Versetzung emittiert werden wird. Diese Behandlung erlaubt die Existenz eines ausgedehnten Versetzungskernes, welches die Notwendigkeit der Kernabschnittradien wie bei dem Rice-Thomson Modell von Emission erübrigt. Ergebnisse werden hier vorgestellt unter mehr realistischen Annahmen für metall-keramische Risse, nämlich die Emission an Gleitebenen innerhalb gemischt-Mode Rißspitzenfeldern, die bezüglich der bestehenden Rißebene schräge sind. Der spezifische Fall von \{221\} Kupferkristallflächen gefügt an Saphir ist analysiert und die Ergebenisse werden angewandt die kürzlichen Beobachtungen von Beltz und Wang [Acta metall. mater. 40, 1675 (1992)] über die richtungsabhängige Zähigkeit an diesen Arten von Grenzflächen zu erklären.

†Present address: Division of Engineering, Brown University, Providence, RI 02912, U.S.A. 


\section{INTRODUCTION}

The issue of the ductile vs brittle response of an interface is treated here, with special emphasis on the emission of dislocations at metal-ceramic interfaces. The aspect examined here is that the ductile vs brittle behaviour of an interface can be partially understood in terms of a competition which occurs on atomic length scales: the emission of dislocations vs cleavage decohesion. (Unless otherwise specified, all discussion concerning the behaviour of dislocations is assumed to be in reference to the metallic part of the interface; it is understood, however, that in certain circumstances the role of dislocation activity in the ceramic may be important also.) A convenient way to parameterize this competition was first introduced in conjunction with some of the later versions $[2-6]$ of the Rice-Thomson model [7], and makes use of the quantities $G_{\text {cleave }}$, the energy release rate for cleavage, and $G_{\text {disl }}$, the energy release rate associated with the emission of a single dislocation on a slip plane emanating from the crack tip. In Fig. 1, the basic premise of the model is illustrated: if $G_{\text {cleave }}<G_{\text {dial }}$, then the crack propagates in a brittle manner, and the interface is said to be intrinsically brittle; conversely, if $G_{\text {dis }}<G_{\text {cleave }}$, then a dislocation is spontaneously emitted thus blunting and "shielding" the crack tip from further increases in applied loading. The energy release rates here should rigorously be interpreted as those based on the local ("screened") crack tip field, which can differ from the macroscopic field due to additional dislocations or other nonlinear effects. The motion of surrounding dislocations in the metal are not accounted for in this treatment, and hence the model is regarded as an oversimplification of the actual crack tip response. Depending on the ease of moving those pre-existing dislocations in the metal, and hence on temperature and loading rate, it may happen that local stresses adequate to meet the cleavage condition $G=G_{\text {cleave }}$ are not attained, even in systems for which $G_{\text {cleave }}<G_{\text {dial }}$, prior to onset of some more ductile fracture mode such as hole growth and/or shearing off.

The primary purpose of this paper is to review the various models of dislocation emission, leading up to the most recent treatment which solves the elasticity problem of a traction-free crack with a Peierls-type stress vs displacement relation being satisfied as a

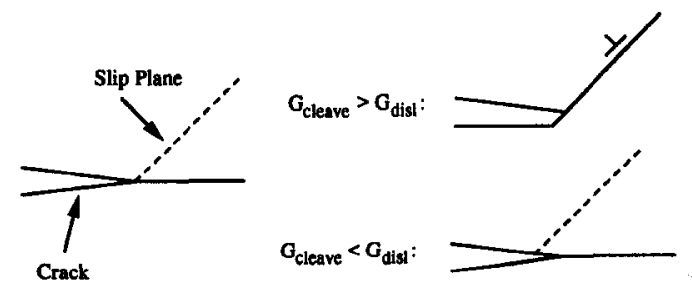

Fig. 1. Atomistically sharp crack on the left, showing the competition between dislocation emission (upper right) and cleavage decohesion (lower right). boundary condition along a slip plane ahead of the crack tip. A review of the experimental effort in this area is also given, which includes work that has centered on the direct observation of dislocation emission from crack tips and work which has successfully utilized Rice-Thomson ideas to predict the directional toughness along interfaces involving copper. Finally, numerical results from the analysis of dislocation nucleation in one such type of specimen are presented; the specimen consists of copper bonded on a $\{221\}$ face to sapphire, a configuration discussed in [6], and has been mechanically tested in recent work by Beltz and Wang [1].

Although the primary focus of this paper is on dislocation emission, a word should be said about cleavage. In the specimens that are discussed later, a critical assumption is that $G_{\text {cleave }}$ is identical at two crack tips which are being compared; it is the difference in $G_{\text {dial }}$ which should give rise to a difference in toughness. The Griffith criterion for crack growth in the absence of plasticity may be used to estimate the energy release rate for cleavage (e.g. $[5,8])$

$$
G_{\text {cleave }}=2 \gamma_{\mathrm{s}}
$$

where $2 \gamma_{\mathrm{s}}$ is twice the surface energy and, and corresponds to the reversible work of fracture. For interfacial fracture, $2 \gamma_{\mathrm{s}}$ generalizes to $2 \gamma_{\text {int }}$, and is given by

$$
2 \gamma_{\text {int }}=\gamma_{s}^{1}+\gamma_{s}^{2}-\gamma_{b}^{1 / 2} .
$$

The parameters $\gamma_{s}^{1}, \gamma_{S}^{2}$ and $\gamma_{b}^{1 / 2}$ correspond to the surface free energies of materials 1 and 2 , and the interfacial free energy prior to separation. A more sophisticated treatment of decohesion is given by the cohesive zone model, which attempts to take into account the non-uniform decohesion that occurs as a crack propagates. In this model, two joined elastic media are initially in contact and decohere within a "transition" zone. A stress vs separation relation $\sigma=\sigma(\delta)$ is assumed to apply along the decohering interface. Application of a well-known $J$-integral calculation gives $[9,10]$

$$
G_{\text {cleave }}=\int_{0}^{\infty} \sigma(\delta) \mathrm{d} \delta=2 \gamma_{\text {int }}
$$

in cases when the decohesion zone is much smaller than the overall crack length $[5,9]$. Note that this is the same result predicted by the Griffith model. Lattice simulations of fracture, in cases for which decohesion is not accompanied by shear-like relaxations between atoms at the crack tip, confirm the Griffith condition [11] except for a modest increase due to lattice trapping.

\section{EXPERIMENTAL OBSERVATIONS}

Dislocation emission from crack tips has been directly observed by the use of several experimental techniques. In work by Burns [12], etch pit techniques were employed to observe edge dislocations on slip planes which emanated from a crack which had been 
cut parallel to the $\{110\}$ planes in lithium fluoride. $\mathrm{X}$-ray topography has been used by Michot and George [13] to carry out similar observations in silicon. Possibly the most notable observations of dislocation emission is the TEM work of Ohr [14, 15], which has the advantage that emission could be observed in situ in several materials, including f.c.c. and b.c.c. metals with a high resolution, typically on the order of a few angstroms. In these experiments, the critical applied stress intensity factor $K_{\mathrm{e}}$ to emit a dislocation was measured; they were in reasonably good agreement for several metals with the theoretical values of $K_{\mathrm{c}}$ as predicted by the Rice-Thomson model. More recently, Chiao and Clark [16] directly observed emitting dislocations in silicon and claimed reasonable agreement of the inferred $K_{e}$ with Rice-Thomson modeling.

The first convincing evidence that the macroscopic behaviour of an interface could be understood based on the competition between dislocation emission and cleavage was given by Wang and Anderson [4], in their work on symmetric tilt bicrystals of copper. In this work, a directional effect on the toughness of the grain boundary in a $\Sigma 9[110](221)$ bicrystal was observed, in which two specimens were cut and notched along the boundary such that a crack would run in the opposite directions [T14] and [114], respectively. The specimens were fatigued under a cyclic mode I loading of increasing amplitude. The specimen with the [1I4] cracking direction broke along the interface when the maximum normal stress reached $\sigma=28.1 \mathrm{MPa}$, corresponding to $G \approx 28 \mathrm{~J} / \mathrm{m}^{2}$. An intergranular fracture surface with cleavage "tongues" was observed. The other specimen, with a cracking direction of [114], was loaded under identical conditions and eventually fractured at a normal stress of $76.7 \mathrm{MPa}$. The fracture surface contained large regions of ductile transgranular fracture and plastic tearing, and the $G$ value, $>210 \mathrm{~J} / \mathrm{m}^{2}$, was beyond the reliably measurable range fracture mechanics. The only difference between these two specimens was the cracking direction, hence it was concluded that the difference in ease with which dislocations could be nucleated at each crack tip was the cause of this behaviour. Further, continuum plasticity analyses by Saeedvafa [17] and Oritz et al. [18], suggested very little difference in the stress state ahead of the crack tip, for the two growth directions, and do not suggest a more macroscopic explanation of the experiments.

Most recently, Beltz and Wang [1] have performed experiments on copper crystals bonded on the same $\{221\}$ face to sapphire, to form a layered beam subjected to four-point bending. Again, a directional dependence of toughness was observed. In their experiment, the ductile direction was observed to be [114], the opposite of the ductile direction with the Wang-Anderson bicrystal specimen. This result was predicted by theory, however, and is elaborated on later in this paper in terms of the Peierls-type nucleation model; it follows from different mode I/II mixity in the two specimens.

\section{EARLY MODELS}

The earliest attempts at quantitatively understanding the factors that control the ductile vs brittle behavior at a crack tip were due to Armstrong [19] and to Kelly et al. [20]. The latter proposed that the response of a crystal or grain boundary should be treated by comparing the ratio of the largest tensile stress to the largest shear stress close to a crack tip with the ratio of the ideal cleavage stress to the ideal shear stress. Armstrong [19] compared the applied stress necessary to meet the Griffith condition with the stress to shear apart a dislocation dipole near a crack tip, and thereby noted the importance of the dimensionless combination $\gamma_{\mathrm{s}} / \mu b\left(\gamma_{\mathrm{s}}=\right.$ surface energy, $\mu=$ shear modulus, $b=$ Burgers vector) as an index of how relatively easy it was for the shear process to occur before cleavage. Subsequently Rice and Thomson [7] modeled the formation and emission of a dislocation from the near-tip region in a (previously) dislocation-free crystal, and considered the ductile vs brittle response of a crystal as a competition between cleavage decohesion and dislocation emission. Their analysis showed, likewise, the importance of large $\gamma_{s} / \mu b$ and also of low core energy (large $r_{\mathrm{c}} / b$, where $r_{\mathrm{c}}$ is the core cut-off radius in their analysis) for ductile response.

As explained in the introduction, recent treatments of the Rice-Thomson model have evolved to characterizing the crack-tip competition in terms of the parameters $G_{\text {clesve }}$ and $G_{\text {disl }}$. In its original form, the Rice-Thomson model treated dislocation emission by two alternate methods; both proceed by assuming the existence of a freshly generated dislocation at a relatively small distance (turning out to be less than a few atomic spacings) away from the crack tip, on a slip plane which intersects the crack front. A drawback to both, as well as the Peierls-type model to be discussed later, is that the analysis may be straightforwardly applied only to cases in which the slip plane(s) intersect the crack front. Following [2], however, we may envision a scenario in which dislocations are emitted when a moving crack front undergoes local deviations which bring it into line with a potentially active slip plane. Another drawback common to both treatments is that they inevitably involve the core cutoff radius, an uncertain parameter [21]; in fact, the basic definition of a dislocation "core" becomes vague as a dislocation is being nucleated.

The first method assumes the dislocation line is straight. Utilizing an elasticity solution for the dislocation in the presence of a crack tip, the force on a dislocation can be determined as a function of distance from the tip and the applied stress intensity factor(s). For a crack on a bimaterial interface, 
consisting of joined isotropic solids, the force is given by Rice et al. [6] as

$$
\begin{aligned}
f= & -\frac{\mu_{1} b^{2}}{4 \pi r}\left[\frac{\cos ^{2} \phi}{1-v_{1}}+\sin ^{2} \phi\right] \\
& +\frac{b}{\sqrt{r}}\left[\operatorname{Re}\left(K r^{i k}\right) S_{1}+\operatorname{Im}\left(K r^{k}\right) S_{\mathrm{II}}+K_{\mathrm{III}} S_{\mathrm{III}}\right]
\end{aligned}
$$

where $r$ is the distance from the tip, $b$ is the length of the Burgers vector, $\phi$ is the orientation of the Burgers vector in the slip plane, and

$$
\begin{aligned}
S_{\mathrm{I}} & =\frac{\sum_{r \theta}^{\mathrm{l}}(\theta) \cos \phi}{\sqrt{2 \pi}}, S_{\mathrm{II}}=\frac{\sum_{r \theta}^{\mathrm{I}}(\theta) \cos \phi}{\sqrt{2 \pi}}, \\
S_{\mathrm{III}} & =\frac{\sum_{2 \theta}^{\mathrm{III}}(\theta) \sin \phi}{\sqrt{2 \pi}} .
\end{aligned}
$$

Here the angular functions $\Sigma_{\alpha \beta}(\theta)$ of superscripts I, II and III appear in the interfacial near-tip stress field, which for dissimilar isotropic materials is given by the form (see $[22,23]$ and references therein)

$$
\begin{aligned}
\sigma_{\alpha \beta}=\frac{1}{\sqrt{2 \pi r}}[\operatorname{Re}( & \left.K r^{k}\right) \Sigma_{\alpha \beta}^{\mathrm{l}}(\theta)+\operatorname{Im}\left(K r^{k}\right) \Sigma_{\alpha \beta}^{\mathrm{II}}(\theta) \\
+ & \left.K_{\mathrm{III}} \Sigma_{\alpha \beta}^{\mathrm{II}}(\theta)\right] \quad(\alpha, \beta=r, \theta, z) .
\end{aligned}
$$

The functions $\Sigma_{\alpha \beta}(\theta)$ correspond to tractions across the interface at $\theta=0$ of tensile, in-plane and antiplane shear type, so that

$$
\left(\sigma_{\theta \theta}+i \sigma_{r \theta}\right)_{\theta=0}=\frac{K r^{i k}}{\sqrt{2 \pi r}}, \quad\left(\sigma_{z \theta}\right)_{\theta=0}=\frac{K_{\mathrm{III}}}{\sqrt{2 \pi r}} .
$$

$K_{\mathrm{III}}$ is the familiar mode III stress intensity factor, and $K$ is the single complex stress intensity factor which characterizes the inherently coupled in-plane modes. The parameter $\epsilon$ is given by

$$
\epsilon=\frac{1}{2 \pi} \ln \left[\frac{\left(3-4 v_{1}\right) / \mu_{1}+1 / \mu_{2}}{1 / \mu_{1}+\left(3-4 v_{2}\right) / \mu_{2}}\right]
$$

where $\mu$ and $\nu$ refer to the shear modulus and Poisson's ratio, respectively. Subscript 1 refers to the material on top, occupying $0<\theta<\pi$, which is taken to be the metal, and subscript 2 refers to the ceramic phase. We have $\Sigma_{\theta \theta}^{\mathrm{I}}(0)=\Sigma_{r \theta}^{\mathrm{II}}(0)=\Sigma_{z \theta}^{\mathrm{III}}(0)=1$ and the full functions $\Sigma_{\alpha \beta}(\theta)$ are given in [6] and can be extracted from discussions of the bimaterial elastic singular field (e.g. [22]).

In this generalization of the first Rice-Thomson approach to interfacial cracks, emission of a dislocation is said to occur when the stress intensity factors are large enough to make $f$ vanish when the position $r$ is equal to one core radius away from the tip, i.e. setting equation (4) equal to zero for $r=r_{c}$ gives the emission criterion. This procedure ensures that the Peach-Koehler force on the dislocation is larger than the image force tending to draw the dislocation back into the tip for all $r>r_{\mathrm{c}}$. In order to carry out this procedure, Rice et al. [6] have taken advantage of the fact that $\boldsymbol{K r}^{\text {ie }}$ is a slowly varying function of $r$. Since the nucleation process typically occurs on length scales of order $b, K r^{k}$ is replaced with $K b^{k}$ when determining the emission criterion. (The real part of $\boldsymbol{K} \boldsymbol{b}^{k}$ must be positive to assure that any predicted material interpenetrations from the oscillating singularity are limited to a subatomic, and hence irrelevant, length scale.) Once the critical stress intensity factors are determined, it is more convenient to express nucleation in terms of an energy release rate. The Irwin-type energy release expression for a bimaterial crack is

$$
G=\left(\frac{1-v_{1}}{\mu_{1}}+\frac{1-v_{2}}{\mu_{2}}\right) \frac{K R}{4 \cosh ^{2} \pi \epsilon}+\left(\frac{1}{\mu_{1}}+\frac{1}{\mu_{2}}\right) \frac{K_{\mathrm{II}}^{2}}{4} .
$$

The final result for in-plane loadings is [6]

$$
\begin{aligned}
& G_{\text {disl }}=\frac{\mu_{1} b^{2}}{\left(1-v_{1}\right)(1-\alpha) r_{\mathrm{c}}} \\
& \times\left[\frac{\cos \phi+\left(1-v_{1}\right) \sin \phi \tan \phi}{4 \sqrt{\pi} \cosh \pi \epsilon\left(\Sigma_{r \theta}^{1}(\theta) \cos \psi^{\prime}+\Sigma_{r \theta}^{\mathrm{II}}(\theta) \sin \psi^{\prime}\right)}\right]^{2}
\end{aligned}
$$

where $\alpha$ is one of the Dundurs parameters, which are given by

$$
\begin{aligned}
& \alpha=\frac{\left(1-v_{2}\right) / \mu_{2}-\left(1-v_{1}\right) / \mu_{1}}{\left(1-v_{2}\right) / \mu_{2}+\left(1-v_{1}\right) / \mu_{1}}, \\
& \beta=\frac{1}{2} \frac{\left(1-2 v_{2}\right) / \mu_{2}-\left(1-2 v_{1}\right) / \mu_{1}}{\left(1-v_{2}\right) / \mu_{2}+\left(1-v_{1}\right) / \mu_{1}} .
\end{aligned}
$$

The quantity $\psi^{\prime}$ is known as the atomic scale phase angle and is the phase angle of $K b^{k}$; it is related to the macroscopic phase angle $\psi$ of $K h^{k}$, associated with macroscopic specimen dimension $h$, by

$$
\psi^{\prime}=\psi-\epsilon \ln (h / b) .
$$

The atomic scale phase angle characterizes the ratio of mode II to mode I loading on atomic length scales from the crack tip.

A second method for determining an emission criterion presupposes the existence of a dislocation loop ahead of a crack tip; the details of this method for bimaterial interfaces have not been worked out. The energy of the loop configuration (in a homogeneous material) may be calculated; it is the sum of contributions from the self energy of the half-loop, the core energy, and the surface energy of the ledge created at the crack tip, less the work done by applied loads to expand the loop to a given radius. For the simple case of mode I loading, the energy is given by $[3,4,7,24]$

$$
\begin{aligned}
E=\pi r\left[A b^{2} \ln \left(\frac{8 m r}{e^{2} b}\right)\right. & \left.+E_{\text {core }}\right] \\
& +2 r E_{\text {ledge }}-3.5 b r^{3 / 2} S K_{\mathrm{I}}
\end{aligned}
$$

where $A=(2-v) \mu / 8 \pi(1-v)$ is the prelogarithmic energy factor and is roughly $10 \%$ of the shear modulus, and $m$ is a constant of order 1-2 [25]. Here $E_{\text {core }}=A b^{2} \ln \left(b / r_{\mathrm{c}}\right)$ and $E_{\text {ledge }}$ is the energy of the ledge left by the crack tip blunting. The critical stress 
intensity factor for emission and radius $r$ at instability are found by $[2,3]$

$$
\frac{\partial E}{\partial r}=0, \quad \frac{\partial^{2} E}{\partial r^{2}}=0
$$

The derivatives are at fixed $K_{1}$; the first condition characterizes equilibrium states ( $r$ and $K_{1}$ pairs) and the second the $K_{\mathrm{I}}$ value at which the energy ceases to be a minimum at a given state (transition from $\partial^{2} E / \partial r^{2}>0$ at lower $K_{\mathrm{I}}$ to $\partial^{2} E / \partial r^{2}<0$ at higher $K_{\mathrm{I}}$ ). The solution for $r$ is, however, typically of the order $b$, whereas equations such as (13) are relatively unambiguous only for $r \gg b$.

Earlier versions of the Rice-Thomson model endeavored to reveal the effect of slip plane orientation and combined $K_{\mathrm{I}}, K_{\mathrm{II}}, K_{\mathrm{III}}$ loading modes on dislocation emission, and to extend the formulation to an interface containing segregated solute atoms. The greatest effect of impurity atoms seems to be on $G_{\text {cleave }}$ through a lowering of $2 \gamma_{\text {int }}$ (for "normal" segregators) [8]. Dislocation emission may also be influenced, however, through a possible solute pinning effect [26], and it is also plausible that some segregants, initially on a crack-containing interface, could segregate along the core of an emerging dislocation and affect nucleation (as discussed in connection with hydrogen by Anderson et al. [27]). The emission of dislocations in dissociated form has been treated extensively in $[3,24]$.

\section{PEIERLS-TYPE NUCLEATION MODEL}

\section{(1) Summary of new approach}

As pointed out in the last section, previous models for calculating $G_{\mathrm{dial}}$ were based on elasticity theory for complete dislocations, and required a knowledge of the core cutoff radius $r_{\mathrm{c}}$ and the core energy $E_{\text {core }}$ $[2,7,24]$. Argon [28] and, more recently, Schöck [29] have recognized that a full dislocation is likely to emerge unstably from an incomplete, incipient dislocation at the tip, but a reasonably exact treatment of the phenomenon has been given only recently [30]. That treatment, reviewed here, solves the elasticity problem of a traction-free crack with a Peierls-type stress vs displacement relation being satisfied as a boundary condition along a slip plane ahead of the crack tip. Once this interfacial "constitutive" relation is specified, and the elasticity problem solved, there is no need for core cut-off parameters. The advantage of this method is that it allows for the existence of an extended dislocation core during nucleation, and eliminates uncertainty involved with choosing the core parameters.

To summarize the new approach [30], assume now the existence of a Peierls-type shear stress $\tau\left(\equiv \sigma_{r \theta}\right.$, on $\theta=0$ in the case now discussed) vs relative atomic displacement (denoted $\Delta_{r}$ ) relation such as the sinusoidal representation in Fig. 2(a); $\Delta_{r}$ denotes the shift of one atomic plane relative to another at the slip (a)

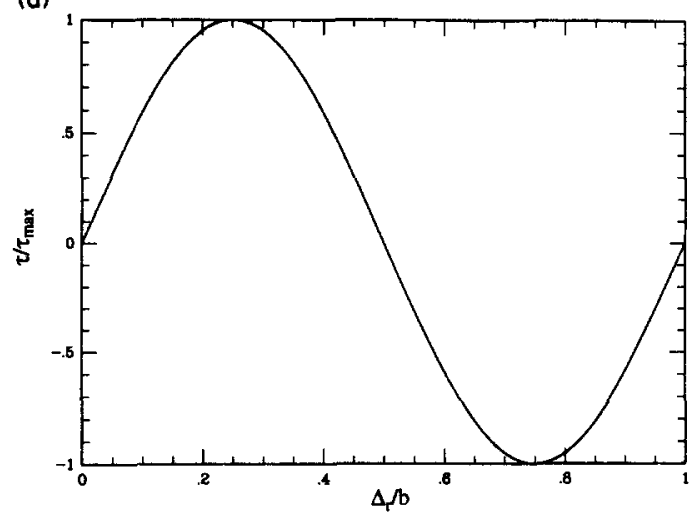

(b)

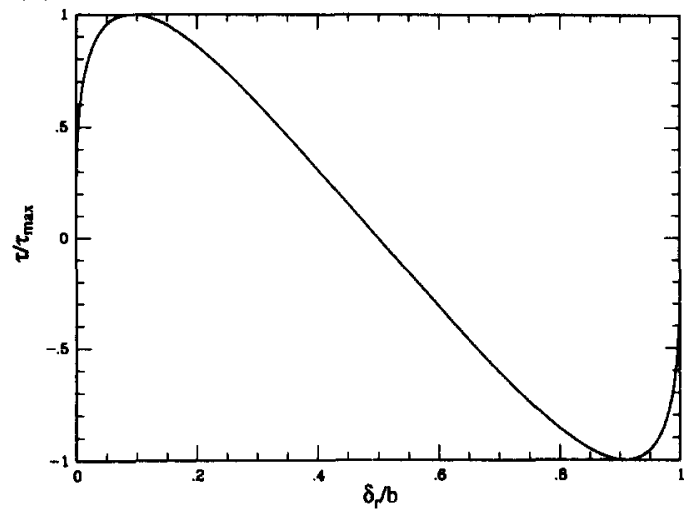

Fig. 2. Expected form of the shear stress $\tau$ vs: (a) relative atomic displacement $\Delta_{\mathrm{r}}$ and (b) displacement discontinuity $\delta_{\mathrm{r}}$.

surface. This curve gives the shear stress needed to locally shear atoms with respect to one another on a given slip plane, and is the fundamental input to the Peierls-Nabarro dislocation model [31,32]. The initial slope of such a curve corresponds with an appropriate shear modulus. The parameter $b$ is the length of a Burgers vector and represents the periodicity of the stress-displacement relation. This type of data has been calculated through the use of pair potentials or the embedded atom method by several researchers $[11,33,34,35]$. The integral of such a curve from $\Delta_{\mathrm{r}}=0$ to the unstable equilibrium position at which the shear stress next vanishes (at $\Delta_{\mathrm{r}}=b / 2$ in simple cases) has been called [30] the unstable stacking energy, denoted $\gamma_{\mathrm{us}}$; the role of this parameter in the dislocation nucleation process is discussed shortly.

Consider a semi-infinite crack subject to mode II loading, in which this Peierls-type stress vs displacement relation is taken to be the constitutive relation on a slip plane which is a coplanar with the crack (see Fig. 3). With the exception of the slip plane, the material is here taken as an isotropic, linear elastic solid with shear modulus $\mu$ and Poisson's ratio $v$; some results, like equations (17) and (18) to follow, do not require those assumptions [30]. Define $\delta_{r}$ as the displacement discontinuity on a mathematical cut 


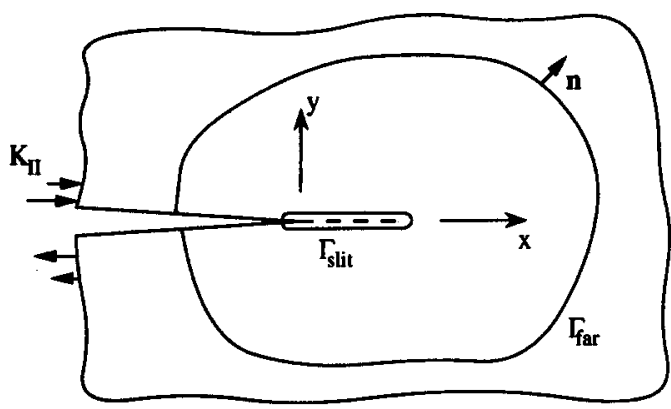

Fig. 3. Geometry for evaluation of the $J$-integral in the far field $\left(\Gamma_{\text {far }}\right)$ and on a path which surrounds the slit which represents a slip plane $\left(\Gamma_{\text {sit }}\right)$.

coincident with the slip plane. We relate $\delta_{r}$ to the displacement $\Delta_{\mathrm{r}}$ of the atomic planes at $\pm h / 2$ from the cut by

$$
\delta_{\mathrm{r}}=u_{\mathrm{r}}^{(+)}-u_{r}^{(-)}=\Delta_{\mathrm{r}}-\frac{\tau h}{\mu}
$$

where $h$ is the interplanar spacing. This idealized cut represents the slip plane, and by adding to the displacement discontinuity $\delta_{\mathrm{r}}$ across the cut (in what is otherwise considered a linear elastic continuum) the additional "elastic" displacement $h \tau / \mu$, we simulate approximately the relative displacement $\Delta_{\mathrm{r}}=\delta_{\mathrm{r}}+h \tau / \mu$ between atomic planes a distance $h$ apart. If $\tau$ is now plotted vs $\delta_{r}$, the curve becomes skewed so as to give an infinite slope at the origin [see Fig. 2(b)]. The integral of $\tau$ over half of a cycle remains equal to $\gamma_{u s}$, however.

In the ensuing calculation, the $J$-integral [10] is used to predict an emission criterion for the situation just discussed, in a manner closely related to its application to tensile decohesion summarized in connection with equation (3). Within this framework, an "incipient dislocation" exist if the function $\delta_{r}(r)$ is nonzero as distance $r$ approaches zero. Evaluation of the $J$-integral on the path $\Gamma_{\text {far }}$ in Fig. 3 gives

$$
J=\frac{1-v}{2 \mu} K_{\mathrm{II}}^{2}=G
$$

when the slit length is much smaller than the crack length (or any other overall length scale associated with the crack-containing elastic body). The quantity $G$ is the energy release rate that would ensue if the crack were to move as a classical singular crack (without a shear or decohesion zone at its tip) quasi-statically under the given value of $K_{\mathrm{II}}$.

Evaluation of the $J$-integral on the path $\Gamma_{\text {slit }}$ (see Fig. 3) gives

$$
J=-\int_{0}^{R_{0}} \tau \frac{\partial \delta_{\mathrm{r}}}{\partial r} \mathrm{~d} r=\int_{0}^{\partial_{\mathrm{tip}}} \tau\left(\delta_{\mathrm{r}}\right) \mathrm{d} \delta_{\mathrm{r}}
$$

where it is assumed that $\delta_{r}(r)$ has decayed effectively to zero for some sufficiently large distance $\boldsymbol{R}_{\mathbf{0}}$ (typically, of order $5 b$ ). The parameter $\delta_{\text {tip }}$ is the displacement evaluated at $r=0$.
If a $\tau$ vs $\delta_{\mathrm{r}}$ curve of the form shown in Fig. 2(b) is assumed, then a plot of $J$ vs $\delta_{\text {tip }}$ would rise monotonically until $\delta_{\text {tip }}=b / 2$, then $J$ would have to decrease to continue along the locus of static solutions, thus giving instability to the atomic configuration under a monotonically increasing external load. The dislocation nucleation criteria is therefore

$$
J_{\mathrm{disl}}=\int_{0}^{b / 2} \tau\left(\delta_{r}\right) \mathrm{d} \delta_{r} \equiv \gamma_{\mathrm{us}} .
$$

More generally, the integral extends to the first value of $\delta_{\mathrm{r}}>0$ at which energy $\Phi=\int \tau \mathrm{d} \delta_{\mathrm{r}}$ has a maximum. Thus, $G=\gamma_{u s}$ is the condition for nucleation of a pure edge dislocation, on a slip plane ahead of the crack tip and parallel to the crack plane; the corresponding $K_{\mathrm{Il}}$ is, from (16) and (18)

$$
K_{\mathrm{II}}^{\text {disl }}=\sqrt{\left(\frac{2 \mu}{1-v}\right) \gamma_{\text {us }}} .
$$

The derivation for a screw dislocation under a mode III loading proceeds in a similar manner, again with the result $G=\gamma_{\mathrm{us}}$ and, because $G=K_{\mathrm{III}}{ }^{2} / 2 \mu$

$$
K_{\mathrm{III}}^{\mathrm{disl}}=\sqrt{2 \mu \gamma_{\mathrm{us}}} \text {. }
$$

Further for combined mode II and mode III loading, and under conditions for which the foregoing $\tau$ vs $\delta_{\mathrm{r}}$ relation is now assumed to apply for slip that is restricted in a direction at angle $\phi$ with the normal to the crack front (so that the final Burgers vector $b$ is oriented at angle $\phi$ ), the result [30] is

$$
\begin{aligned}
& K_{\mathrm{II}}^{\text {disl }} \cos \phi+K_{\mathrm{III}}^{\text {disl }} \sin \phi \\
& \quad=\sqrt{\left(\frac{2 \mu}{1-v}\right)\left[\cos ^{2} \phi+(1-v) \sin ^{2} \phi\right] \gamma_{\mathrm{us}}} .
\end{aligned}
$$

Complications arise, however, when we include in the model the effects of normal tractions and dilatant opening across the slip plane. This situation occurs if a mode I-type loading is added to the mode II situation just discussed, or in more realistic cases when the slip plane is inclined with respect to the crack plane. There are no reasons to assume that a given $\tau$ vs $\delta_{\mathrm{r}}$ curve retains its shape if tension is superposed; hence the effect of superposed tension on the "effective" $\gamma_{u s}$ must be investigated. Argon [28] and Cheung et al. [36] have already noted the importance of softening in shear due to large tensile stresses across a slip plane.

\section{(2) Combined tension and shear relations}

Let $\Delta_{\theta}$ be the relative atomic separation across the slip plane (see Fig. 4). Analogous to the case for shearing, a suitable displacement variable for the mathematical cut representing the slip plane may be defined

$$
\delta_{\theta}=u_{\theta}^{(+)}-u_{\theta}^{(-)}=\Delta_{\theta}-\frac{L^{2} \sigma}{2 \gamma_{\mathrm{S}}} .
$$

The quantity $2 \gamma_{s} / L$ is an effective Young's modulus for tension across the slip plane and corresponds to 


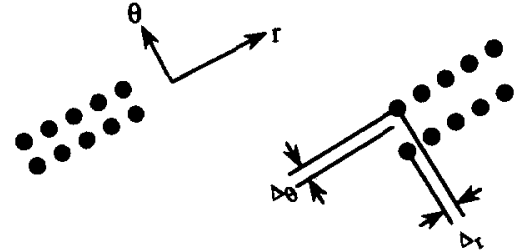

Fig. 4. Schematic showing the measures of relative atomic displacements in the $r$ (shear) and $\theta$ (opening) directions.

the initial slope of the $\sigma\left(\equiv \sigma_{\theta \theta}\right)$ vs $\Delta_{\theta}$ relation to be discussed shortly [8]. Here a particular form of the $\tau$ vs $\Delta_{\mathrm{r}}$ and $\sigma$ vs $\Delta_{\theta}$ relations are chosen for the purpose of solving some particular problems. Assume that the curve in Fig. 2(a) is a sinusoid (commonly known as the Frenkel sinusoid [30])

$$
\tau=\frac{\pi \gamma_{\mathrm{us}}}{b} \sin \left(\frac{2 \pi \Delta_{\mathrm{r}}}{b}\right)
$$

where the amplitude factor is chosen such that

$$
\int_{0}^{b / 2} \tau\left(\Delta_{\mathrm{r}}\right) \mathrm{d} \Delta_{\mathrm{r}}=\gamma_{\mathrm{us}} \text {. }
$$

Requiring, as in the discussion of equation (15), that $\mathrm{d} \tau / \mathrm{d}\left(\Delta_{\mathrm{r}} / h\right)=\mu$ when $\Delta_{\mathrm{r}}=0$ then shows that $\gamma_{\mathrm{us}}=\mu b^{2} / 2 \pi^{2} h$ when the Frenkel form is adopted. Here we take the sinusoid to be the proper form for $\tau$ when $\Delta_{\theta}$ is zero, understanding that the $\gamma_{u s}$ which enters it is that for unrelaxed shear. The corresponding relation for tensile decohesion is assumed to be

$$
\sigma=\frac{2 \gamma_{\mathrm{S}}}{L^{2}} \Delta_{\theta} \mathrm{e}^{-\Delta_{\theta} / L}
$$

when $\Delta_{\mathrm{r}}=0$. This is an example of a stress vs separation relation as discussed in connection with equation (3), and follows from the well-known fit, with energy proportional to $-\left(L+\Delta_{\theta}\right) \exp \left(-\Delta_{\theta} / L\right)$, to the universal bonding correlation of Rose et al. [37-39]. The parameter $L$ has been suggested as scaling with the Thomas-Fermi screening length; here it can be loosely interpreted as the characteristic length associated with the decohesion process ( $\sigma$ reaches its maximum, at $\Delta_{\mathrm{r}}=0$, when $\Delta_{\theta}=L$ ). The constants on the right-hand side are chosen to enforce

$$
\int_{0}^{\infty} \sigma\left(\Delta_{\theta}\right) \mathrm{d} \Delta_{\theta}=2 \gamma_{s}
$$

as required by the definition of $2 \gamma_{\mathrm{s}}$.

For general loadings the functions $\tau\left(\Delta_{\mathrm{r}}, \Delta_{\theta}\right)$ and $\sigma\left(\Delta_{r}, \Delta_{\theta}\right)$ must be derivable from a potential function which corresponds to the energy per unit area on the slip plane through the relations

$$
\tau=\frac{\partial \Psi}{\partial \Delta_{\mathrm{r}}}, \quad \sigma=\frac{\partial \Psi}{\partial \Delta_{\theta}}
$$

which are equivalent to the requirement

$$
\frac{\partial \sigma}{\partial \Delta_{\mathrm{r}}}=\frac{\partial \tau}{\partial \Delta_{\theta}}
$$

We now proceed by assuming the following generalized forms for $\tau\left(\Delta_{r}, \Delta_{\theta}\right)$ and $\sigma\left(\Delta_{r}, \Delta_{\theta}\right)$ involving functions $A\left(\Delta_{\theta}\right), B\left(\Delta_{\mathrm{r}}\right)$ and $C\left(\Delta_{\mathrm{r}}\right)$ to be determined

$$
\begin{aligned}
& \tau=A\left(\Delta_{\theta}\right) \sin \left(\frac{2 \pi \Delta_{\mathrm{r}}}{b}\right) \\
& \sigma=\left[B\left(\Delta_{\mathrm{r}}\right) \Delta_{\theta}-C\left(\Delta_{\mathrm{r}}\right)\right] \mathrm{e}^{-\Delta_{\theta} / L} .
\end{aligned}
$$

Enforcing equations (24), (26) and (28) with $C(0)$, and requiring that $\tau$ and $\sigma$ vanish as $\Delta_{\theta}$ becomes unbounded, leads to the following as the most general possible expressions for functions $A, B$ and $C$

$$
\begin{aligned}
A\left(\Delta_{\theta}\right) & =\frac{\pi \gamma_{\mathrm{us}}}{b}-\frac{2 \pi \gamma_{\mathrm{S}}}{b} \\
\times & \left\{q\left(1-\mathrm{e}^{-\Delta_{\theta} / L}\right)-\left(\frac{q-p}{1-p}\right) \frac{\Delta_{\theta}}{L} \mathrm{e}^{-\Delta_{\theta} / L}\right\} \\
B\left(\Delta_{\mathrm{r}}\right) & =\frac{2 \gamma_{\mathrm{s}}}{L^{2}}\left\{1-\left(\frac{q-p}{1-p}\right) \sin ^{2}\left(\frac{\pi \Delta_{\mathrm{r}}}{b}\right)\right\} \\
C\left(\Delta_{\mathrm{r}}\right) & =\frac{2 \gamma_{\mathrm{s}}}{L} \frac{p(1-q)}{1-p} \sin ^{2}\left(\frac{\pi \Delta_{\mathrm{r}}}{b}\right)
\end{aligned}
$$

where

$$
q=\frac{\gamma_{\mathrm{us}}}{2 \gamma_{\mathrm{s}}}, \quad p=\frac{\Delta_{\theta}^{*}}{L}
$$

and where $\Delta_{\theta}^{*}$ is the value of $\Delta_{\theta}$ after shearing to the state $\Delta_{\mathrm{r}}=b / 2$ under conditions of zero tension, $\sigma=0$ (i.e. relaxed shearing). The parameter $p$ is referred to here as the "dilation parameter." It is possible to obtain an explicit form for $\Psi$ by combining equations (29) and (30) and then integrating; in fact when $q=p$, it is the same form used by Needleman [40] in conjunction with the analysis of the decohesion of a viscoplastic block from a rigid substrate. Embedded atom method fits to material properties have been employed to estimate $L / b, q$ and $p$; the results of two such studies $[34,35]$ are used as a guideline for estimating the parameters used in this investigation. Recent estimates suggests that $q$ ranges from 0.05 to 0.08 in f.c.c. materials, assuming a dislocation is emitted as a pair of partials [34]. Estimates for b.c.c. materials, based on results in $[11,35,41]$, show that $q$ is about 0.12 for iron and may be as large as 0.3 for others.

\section{(3) Solutions involving inclined slip planes}

Consider again a semi-infinite crack on an interface consisting of joined isotropic materials, with material 1 on top. Let a slip plane emanate from the crack tip at some angle $\theta$ with respect to the crack plane (see Fig. 5); this plane is modelled as a semi-infinite mathematical cut which obeys the constitutive relations developed in the previous section. Without loss of generality, we may restrict the cut to reside within material 1 . Let $r$ denote the distance from the crack tip along the cut. Assume that the loading is in-plane; hence equilibrium may be imposed across 


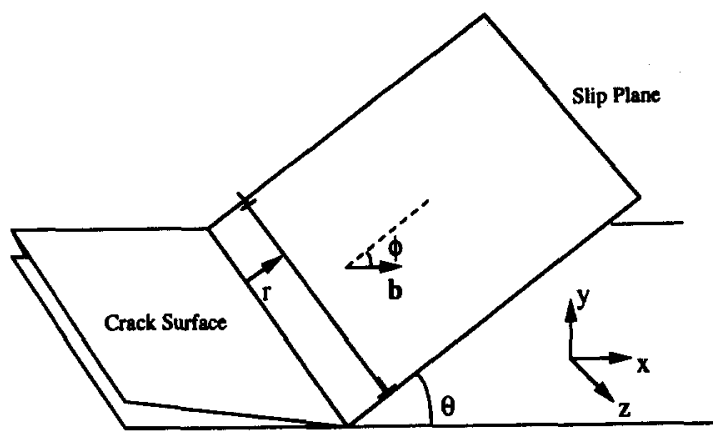

Fig. 5. Geometry used for the analysis of dislocation emission on an inclined slip plane.

the slip plane for a given complex stress intensity factor $K$

$$
\begin{aligned}
\tau\left[\delta_{\mathrm{r}}(r), \delta_{\theta}(r)\right]= & \frac{1}{\sqrt{2 \pi r}}\left\{\operatorname{Re}\left[K r^{k}\right] \Sigma_{r \theta}^{\mathrm{I}}(\theta ; \epsilon)\right. \\
& \left.+\operatorname{Im}\left[K r^{\natural}\right] \Sigma_{r \theta}^{\mathrm{II}}(\theta ; \epsilon)\right\} \\
& -\frac{\mu_{1}}{2 \pi\left(1-v_{1}\right)} \int_{0}^{\infty} g_{11}(r, s) \frac{\partial \delta_{\mathrm{r}}}{\partial s} \mathrm{~d} s \\
& -\frac{\mu_{1}}{2 \pi\left(1-v_{1}\right)} \int_{0}^{\infty} g_{12}(r, s) \frac{\partial \delta_{\theta}}{\partial s} \mathrm{~d} s \\
\sigma\left[\delta_{\mathrm{r}}(r), \delta_{\theta}(r)\right]= & \frac{1}{\sqrt{2 \pi r}}\left\{\operatorname{Re}\left[K r^{i}\right] \Sigma_{\theta \theta}^{\mathrm{I}}(\theta ; \epsilon)\right. \\
& \left.+\operatorname{Im}\left[K r^{k}\right] \Sigma_{\theta \theta}^{\mathrm{II}}(\theta ; \epsilon)\right\} \\
& -\frac{\mu_{1}}{2 \pi\left(1-v_{1}\right)} \int_{0}^{\infty} g_{21}(r, s) \frac{\partial \delta_{\mathrm{r}}}{\partial s} \mathrm{~d} s \\
& -\frac{\mu_{1}}{2 \pi\left(1-v_{1}\right)} \int_{0}^{\infty} g_{22}(r, s) \frac{\partial \delta_{\theta}}{\partial s} \mathrm{~d} s .
\end{aligned}
$$

The first term on the right hand side of each integral equation is the linear elastic contribution to the stress, given by the asymptotic interfacial crack tip field; the second term in each is due to the nonlinear shear and opening displacements along the cut, which is modelled as a continuous distribution of infinitesimal dislocations. The kernel functions $g_{11}, g_{12}, g_{21}$ and $g_{22}$ are taken from the elasticity solution for a dislocation in the presence of an interfacial crack, and may be found in complex form in [42]. Combining with equation (9), so that the equations are expressed in terms of the applied energy release rate and the atomic scale phase angle gives

$$
\begin{aligned}
\tau\left[\delta_{\mathrm{r}}(r), \delta_{\theta}(r)\right]= & \cosh \pi \epsilon \sqrt{\frac{G E^{*}}{2 \pi r}}\left\{\operatorname{Re}\left[\mathrm{e}^{\mathrm{N} \psi^{\prime}}(r / b)^{i}\right] \Sigma_{r \theta}^{\mathrm{I}}(\theta)\right. \\
& \left.+\operatorname{Im}\left[\mathrm{e}^{\omega^{\prime}}(r / b)^{k}\right] \Sigma_{r \theta}^{\mathrm{II}}(\theta)\right\} \\
& -\frac{\mu_{1}}{2 \pi\left(1-v_{1}\right)} \int_{0}^{\infty} g_{11}(r, s) \frac{\partial \delta_{\mathrm{r}}}{\partial s} \mathrm{~d} s \\
& -\frac{\mu_{1}}{2 \pi\left(1-v_{1}\right)} \int_{0}^{\infty} g_{12}(r, s) \frac{\partial \delta_{\theta}}{\partial s} \mathrm{~d} s
\end{aligned}
$$

$$
\begin{aligned}
\sigma\left[\delta_{\mathrm{r}}(r), \delta_{\theta}(r)\right]= & \cosh \pi \epsilon \sqrt{\frac{G E^{*}}{2 \pi r}}\left\{\operatorname{Re}\left[\mathrm{e}^{h \psi^{\prime}}(r / b)^{k}\right] \Sigma_{\theta \theta}^{\mathrm{I}}(\theta)\right. \\
& \left.+\operatorname{Im}\left[\mathrm{e}^{i \psi^{\prime}}(r / b)^{k}\right] \Sigma_{\theta \theta}^{\mathrm{II}}(\theta)\right\} \\
& -\frac{\mu_{1}}{2 \pi\left(1-v_{1}\right)} \int_{0}^{\infty} g_{21}(r, s) \frac{\partial \delta_{\mathrm{r}}}{\partial s} \mathrm{~d} s \\
& -\frac{\mu_{1}}{2 \pi\left(1-v_{1}\right)} \int_{0}^{\infty} g_{22}(r, s) \frac{\partial \delta_{\theta}}{\partial s} \mathrm{~d} s .
\end{aligned}
$$

The integration variable $s$ also denotes distance from the crack tip along the slip plane, and $1 / E^{*}$ is the average of $(1-v) / 2 \mu$.

Equation (33) may be solved numerically for particular values of $G, \psi^{\prime}$ and $\theta$ by mapping the region $0<r<\infty$ onto the domain $-1<\eta<+1$ (and likewise for $s$ ) via the transformations

$$
r=b \frac{1+\eta}{1-\eta}, \quad s=b \frac{1+\xi}{1-\xi} .
$$

The domains of $\eta$ and $\xi$ are then discretized and the coupled integral equations (33) are pointwise enforced at values of $\eta$ given by

$$
\eta_{i}=\cos \frac{\pi(2 i-1)}{2(n+1)}, \quad i=1, \ldots, n+1 \text {. }
$$

Integration is carried out by discretizing the domain of $\xi$ in the following manner

$$
\xi_{j}=\cos \frac{j \pi}{n+1}, j=1, \ldots, n
$$

and making use of the Gauss-Chebyshev integration formulae [43]. We used $n=\mathbf{4 0}$ for the results noted here. The resulting set of nonlinear algebraic equations are solved by choosing a low value of $G$, and iterating to convergence with the Newton-Raphson method. In most cases, convergence is obtained after only two or three iterations. The applied $G$ is then incremented, and the solution procedure is repeated using the previous solution as the initial guess. As solutions are obtained for increasing $G$, the determinant of the Jacobian matrix $\mathbf{J}$ of the nonlinear algebraic equations is monitored. In all cases observed, this parameter rapidly decreases towards zero in a narrow range of $G$ (see [44] for examples). When this determinant is zero, the equations become singular and hence no solution is possible. The sharp decrease of det $\mathbf{J}$ is interpreted here as the onset of instability, i.e. the emission of the dislocation, and the solution procedure is halted.

\section{(4) Applications to single crystal Cu/sapphire inter- faces}

The specimen analyzed here is a four-point bend specimen consisting of a single crystal of copper bonded to a thinner sapphire layer (see Fig. 6). The sapphire layer is cracked to the interface and a central crack continues along the interface in two opposing directions. This type of specimen was originally analyzed by Charalambides et al. [45] and Suo and 

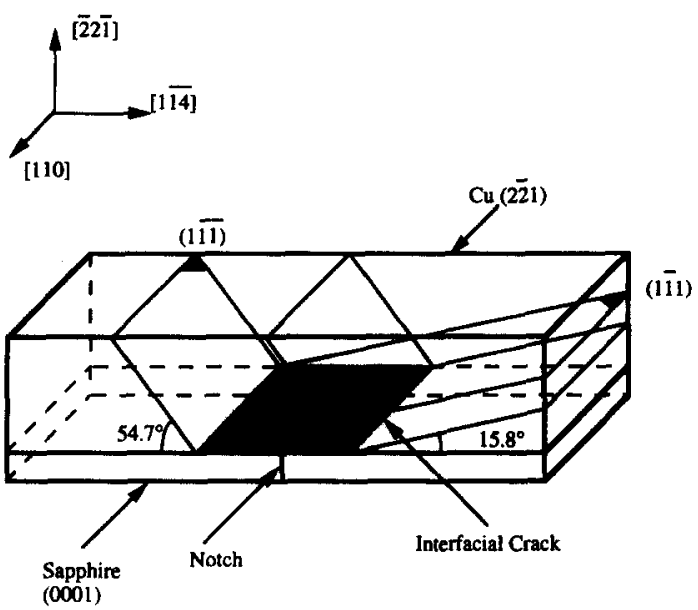

Fig. 6. Diagram of the specimen analyzed: a copper single crystal with $\{221\}$ face bonded to sapphire; loaded in bending with crack tips along [110].

Hutchinson [46] for the testing of linear elastic interfacial fracture mechanics concepts; the usefulness of the specimen for checking a possible directional effect resulting from the use of an asymmetrically aligned metal single crystal was pointed out in [6]. An advantage to this type of specimen is that when the crack is long compared with the thickness of the notched layer $h$, the stress intensity factor has the following form, which is independent of crack length

$$
K=Y(\alpha, \beta, h / H) M h^{-3 / 2-i \epsilon} \mathrm{e}^{i \psi(\alpha, \beta, h / H)}
$$

where $M$ is the applied moment per unit length, and $Y$ and $\psi$ are dimensionless functions of the thickness ratio and the Dundurs parameters $\alpha$ and $\beta$. The functions $Y$ and $\psi$ may be found in [42] and [46].

The intent of the four-point bend specimen is to compare crack growth in two opposing directions in the same specimen during the same mechanical test. The orientation of the copper analyzed here is the same as that used in the experiments of Wang and Anderson [4] and Beltz and Wang [1]: the (2₹1) face of the copper crystal is bonded to the sapphire surface, with the short dimension of the interface parallel to the [110] direction in the copper. Once a crack runs through the sapphire and branches onto the interface in both directions, the crack fronts at both ends lie along the intersection of a pair of $\{111\}$ slip planes of the copper crystal with the interface. The crack growth directions are hence [T14] and [114]. Because of the asymmetry of the copper crystal, the crack oriented to run in the [114] direction encounters slip planes which are aligned at $15.8^{\circ}$ and $125.3^{\circ}$, respectively, while the crack oriented to run in the [T14] direction encounters slip planes inclined at $54.7^{\circ}$ and $164.2^{\circ}$.

As discussed in [6], the atomic scale phase angle for this type of $\mathrm{Cu} / \mathrm{sapphire}$ specimen and the type of loading under consideration is approximately $-79^{\circ}$, and thus involves a very substantial mode II component. Although two possible slip planes are avail- able at each crack tip, it has been shown $[6,47]$ that dislocation emission in this type of specimen is possible only on the slip planes at $125.3^{\circ}$ for the crack which runs in the [114] direction and $164.2^{\circ}$ for the crack which runs in the [T14] direction. The other two slip planes have resolved shear stresses which favor dislocation motion towards the crack tip, causing the crack walls to be driven together, and hence are uninteresting cases. The solution procedure described in the preceding section was hence carried out for $\Psi^{\prime}=-79^{\circ}, \theta=125.3$ and $164.2^{\circ}$, and other elastic constants appropriate to copper and sapphire. The parameter $q$ was taken as 0.08 and $p$ was taken as 0.0 and 0.1 ; the latter value of $p$ leads to a higher degree of coupling in the stress vs displacement relations, but does not affect the general conclusions of this study. We neglect elastic anisotropy of the joined solids, as for the simplified analysis outlined above.

\section{NUMERICAL RESULTS}

In Figs 7(a) and 8, the applied energy release rate $G / \gamma_{\mathrm{w}}$ is plotted as a function of the crack tip opening displacement $\delta_{\mathrm{r}}^{\mathrm{tip}} / \boldsymbol{b}$ for the two angles of interest. In these figures, the $\gamma_{\mathrm{us}}$ is that for unrelaxed shear. Here $b$ is the Burgers vector of the partial (i.e. $b_{\text {total }} / \sqrt{3}$ ). When $\theta=125.3^{\circ}$ (i.e. crack growth in the [114]
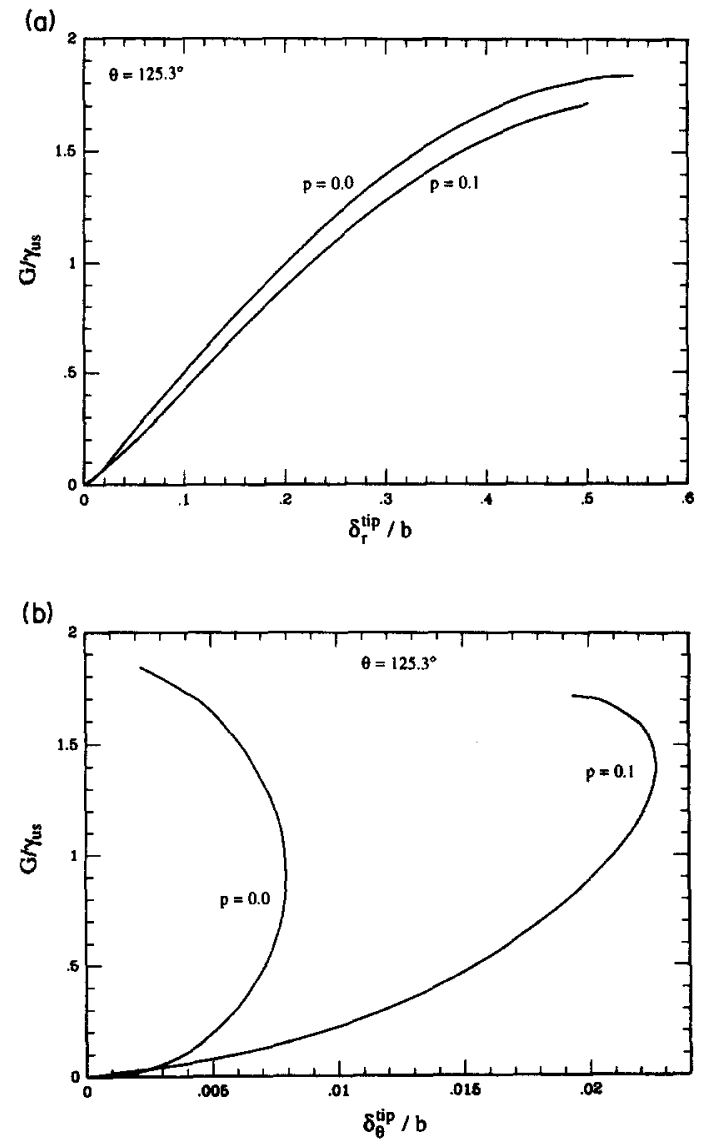

Fig. 7. The applied energy release rate vs: (a) $\delta_{\mathrm{f}}^{\text {tip }}$ and (b) $\delta_{\theta}^{\text {up }}$ for the two values of $p$; slip plane at $\theta=125.3^{\circ}$. 


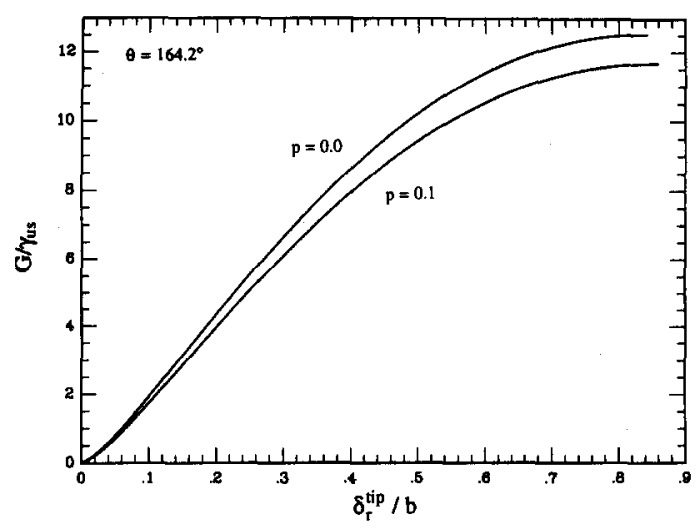

Fig. 8. The applied energy release rate vs $\delta_{\mathrm{f}}^{\text {tip }}$ for the two values of $p$; slip plane at $\theta=164.2^{\circ}$.

direction), unstable nucleation of the first partial occurs at $G / \gamma_{\mathrm{us}}=1.839$ for $p=0$, and instability occurs at $G / \gamma_{\text {us }}=1.715$ for $p=0.1$. When $\theta=164.2^{\circ}$ (crack growth in the [T14] direction), unstable nucleation occurs at $G / \gamma_{\mathrm{us}}=12.55$ for $p=0$ and at $G / \gamma_{\mathrm{us}}=11.69$ when $p=0.1$. There is more than a factor of 6 difference in $G_{\text {disl }}$ for crack growth in the opposing directions (regardless of $p$ ); hence, it is concluded that dislocation nucleation is preferred in [1T4] direction, and blunting should be favored in this growth direction. This result is consistent with the experimental observations of Beltz and Wang [1] on this type of interface. It is the substantial mode II/mode I ratio here which renders as ductile the direction which was brittle in Wang and Anderson's [4] mode I loaded bicrystal, and vice versa.

At instability, the shear displacments at the crack tip are greater than $b / 2$, and this effect is more marked for the case of $\theta=164^{\circ}$. Recall, however, that the result that $\delta_{\mathrm{r}}^{\text {tip }} / \mathrm{b}=0.5$ is only valid for the simple case of a mode II shear crack in a homogeneous material. More importantly, note that the plots of $G / \gamma_{\text {us }}$ vs $\delta_{\mathrm{r}}^{\mathrm{tip}} / b$ (with one exception) become locally flat at instability, indicating a local maximum in $G / \gamma_{u s}$. In the case when $\theta=125.3^{\circ}$ and $p=0.1$, the results for $G / \gamma_{\text {us }}$ vs $\delta_{\theta}^{\text {tip }} / b$ [see Fig. 7(b)]

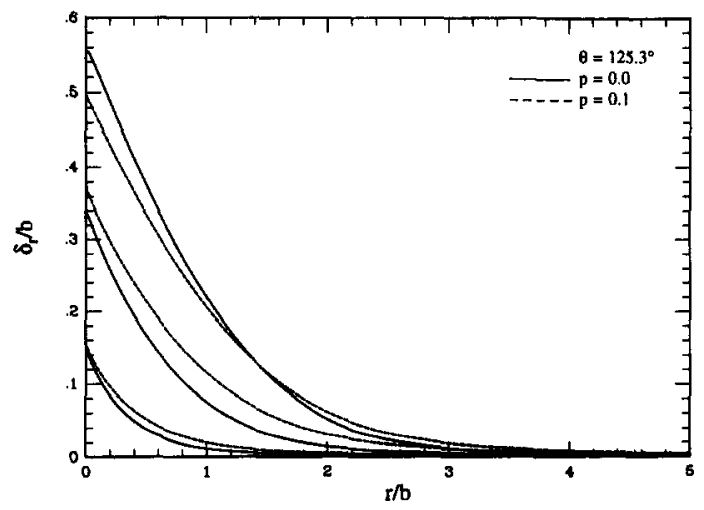

Fig. 9. Displacement profiles for $\theta=125.3^{\circ}$, at various load levels up to instability.

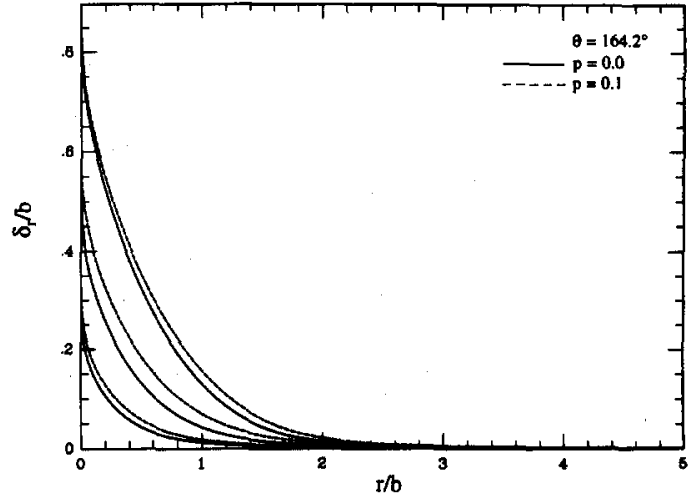

Fig. 10. Displacement profiles for $\theta=164.2^{\circ}$, at various load levels up to instability.

illustrate the possibility that instability may also be associated with opening along the slip planes rather than shearing; this follows from the fact that $G / \gamma_{\mathrm{us}}$ never reaches a maximum with respect to $\delta_{\mathrm{r}}^{\mathrm{tip}} / b$, but does reach a local maximum with respect to $\delta_{\theta}^{\text {tip }} / b$ (despite the fact that $\delta_{\theta}^{\text {tip }} / b$ begins to diminish prior to instability).

In Figs 9 and 10, the shear displacement profiles are given for various levels of applied load up to instability; these figures dramatically illustrate the "birth" of a dislocation. The characteristic length over which $\delta_{\mathrm{r}}$ is appreciable is roughly $(2-3) b$. This length gives a measure of the "halfwidth" of the incipient edge dislocation that exists at the crack tip. If the Peierls-Nabarro dislocation model is applied to a single edge dislocation (not in the presence of a crack tip) and halfwidth is given by $h / 2(1-v)$, where $h$ is the interplanar spacing [48]. This quantity is arbitrarily defined as the distance over which $b / 4<\Delta_{\mathrm{r}}<b / 2$, and is equal to about $0.75 b$ for the isolated dislocation (assuming $b=h$, to be consistent with the way the equations were set up in this study). Applying the same definition to the incipient dislocation gives a width of about $2 b$. The fact that the dislocation core seems to be wider prior to emission adds more uncertainty to the Rice-Thomson equations for nucleation, which make use of a core cut-off concept. The greater width also makes use of the Peierls-Nabarro concept yet more applicable for dislocation nucleation from a crack tip than it is for a dislocation in an otherwise perfect crystal.

The specific results shown apply for nucleation of one of two partial dislocations for the copper crystal orientation considered. Whereas the second partial nucleates at $\phi=0^{\circ}$, the first does so at $\phi=60^{\circ}$. Our numerical procedures are not yet adequate to deal with the latter case, but an elementary estimate based on equation (21) from [30] with $\phi=60^{\circ}$ suggests that each $G$ will increase by a factor of $(4-3 v) \approx 3$ to nucleate the second partial, thus retaining the factor of 6 difference between nucleation levels for the two cracking directions considered. 


\section{SUMMARY}

An improved, Peierls-type model has been presented for modelling the nucleation of dislocations from cracks along interfaces. The advantage of the model is that nonlinear effects which are inherently associated with extended dislocation cores are realistically handled. The results justify this approach, since the incipient dislocations analyzed here displayed larger cores than when present in a perfect crystal. Predictions of the model for copper/sapphire interfaces are in general agreement with experimental observations on bend specimens consisting of copper bonded on a $\{221\}$ face to sapphire. The agreement indicates that the different fracture behaviour of metal-ceramic interfaces may be understood, at least qualitatively, by comparing the values of crack tip energy release rate for dislocation emission from the crack tip against that for cleavage decohesion of the boundary.

Acknowledgements-This research is supported by the NSF Materials Research Laboratory at Harvard (grant DMR89-20490) and a University Research Initiative (subcontract POAVB38639-0 from the University of California, Santa Barbara, based on ONR/DARPA contract N00014-86-K0753). Some of the computations were carried out under NSF support at the Pittsburgh Supercomputing Center. We are grateful to Y. Cui, I.-H. Lin, Y. Sun, and J.-S. Wang for helpful discussions.

\section{REFERENCES}

1. G. E. Beltz and J.-S. Wang, Acta metall. mater. 40, 1675 (1992).

2. D. Mason, Phil. Mag. 39, 455 (1979).

3. P. M. Anderson and J. R. Rice, Scripta metall. 20, 1467 (1986).

4. J.-S. Wang and P. M. Anderson, Acta metall. mater. 39, 779 (1991).

5. J. R. Rice, in Chemistry and Physics of Fracture (edited by $R$. M. Latanision and R. H. Jones), p. 22. Martinus Nijhoff, Dordrecht (1987)

6. J. R. Rice, Z. Suo and J.-S. Wang, in Metal-Ceramic Interfaces (edited by M. Rühle, A. G. Evans, M. F. Ashby, and J. P. Hirth), pp. 269-294. Pergamon Press, Oxford (1990).

7. J. R. Rice and R. Thomson, Phil. Mag. 29, 73 (1974).

8. J. R. Rice and J. S. Wang, Mater Sci. Engng A 107, 23 (1989).

9. J. R. Rice, in Fracture: An Advanced Treatise (edited by H. Liebowitz), Vol. II, p. 191. Academic Press, New York (1968).

10. J. R. Rice, J. appl. Mech. 35,379 (1968).

11. K. Cheung, Ph.D. thesis, Massachusetts Institute of Technology, Cambridge, Mass. (1990).

12. S. J. Burns, Scripta metall. 20, 1489 (1986).

13. G. Michot and A. George, Scripta metall. 20, 1495 (1986).

14. S. M. Ohr, Mater Sci. Eng. 72, 1 (1985).

15. S. M. Ohr, Scripta metall. 20 , 1501 (1986).
16. Y.-H. Chigo and D. R. Clark, Acta metall. 37, 203 (1989).

17. M. Sacedvafa, Mech. Mater., submitted.

18. R. Mohan, M. Ortiz, and C. F. Shih, in Modeling the Deformation of Crystalline Solids (edited by T. C. Lowe, A. D. Rollett, P. S. Follansbee and G. S. Daehn), p. 481. TMS (1991)

19. R. W. Armstrong, Mater. Sci. Engng 1, 251 (1966).

20. A. Kelly, W. R. Tyson and A. H. Cottrell, Phil. Mag. 15, 567 (1967).

21. F. Prinz, H. O. K. Kirchner and G. Schoeck, Phil. Mag. 38, 321 (1978).

22. J. R. Rice, J. appl. Mech. 55, 98 (1988).

23. J. W. Hutchinson, in Metal-Ceramic Interfaces (edited by M. Rühle, A. G. Evans, M. F. Ashby, and J. P. Hirth), pp. 295-306. Pergamon Press, Oxford (1990).

24. P. M. Anderson, Ph.D. thesis, Harvard Univ., Cambridge, Mass. (1986).

25. H. Gao and J. R. Rice, J. Mech. Phys Solids. 37, 155 (1989).

26. J. Yu and J. R. Rice, in Interfacial Structure, Properties, and Design (edited by M. H. Yoo, C. L. Briant and W. A. T. Clark), Vol. 122, p. 361. MRS, Pittsburgh, Pa (1988).

27. P. M. Anderson, J.-S. Wang and J. R. Rice, in Innovations in Ultrahigh-Strength Steel Technology, Sagamore Army Materials Research Conference Procedings, Vol. 34 (edited by G. B. Olson, M. Azrin and E. S. Wright), p. 619. U.S. Army Materials Technology Laboratory, Watertown, Mass. (1990).

28. A. S. Argon, Acta metall, 35, 185 (1987).

29. G. Schöck, Phil. Mag. A 63, 111 (1991).

30. J. R. Rice, J. Mech. Phys. Solids 40, 239 (1992).

31. R. E. Peierls, Proc. phys. Soc. 52, 23 (1940).

32. F. R. N. Nabarro, Proc. phys. Soc. 59, 59 (1947).

33. M. Yamaguchi, V. Vitek and D. P. Pope, Phil. Mag. 43, 1027 (1981)

34. Y. Sun, J. R. Rice and L. Truskinovsky, in HighTemperature Ordered Intermetallic Alloys (edited by L. A. Johnson, D. T. Pope and J. O. Stiegler), Vol. 213. MRS, Pittsburgh, PA (1991).

35. Y. Sun, G. E. Beltz and J. R. Rice, manuscript in preparation.

36. K. S. Cheung, A. S. Argon and S. Yip, J. appl. Phys. 69, 2088 (1991)

37. J. H. Rose, J. R. Smith and J. Ferrante, Phys. Rev. B 28, 1835 (1983).

38. J. H. Rose, J. R. Smith, F. Guinea and J. Ferrante, Phys. Rev. B 29, 2963 (1984).

39. J. Ferrante and J. R. Smith, Phys. Rev. B 31, 3427 (1985).

40. A. Needleman, J. Mech. Phys. Solids, 38, 289 (1990).

41. A. T. Paxton, P. Gumbsch and M. Methfessel, Phys. Mag. Lett. 63, 267 (1991).

42. Z. Suo, Ph.D. thesis, Harvard Univ., Cambridge, Mass. (1989).

43. F. Erodogan and G. D. Gupta, Q. appl. Math. 29, 525 (1972).

44. G. E. Beltz and J. R. Rice, in Modeling the Deformation of Crystalline Solids (edited by T. C. Lowe, A. D. Rollet, P. S. Follansbee and G. S. Daehn), p. 457. TMS (1991).

45. P. G. Charalambides, J. Lund, A. G. Evans and R. M McMeeking, J. appl. Mech. 56, 77 (1989).

46. Z. Suo and J. W. Hutchinson, Int. J. Fract. 43, 1 (1990).

47. G. E. Beltz, unpublished work.

48. J. P. Hirth and J. Lothe, Theory of Dislocations, 2nd edn. Wiley, New York (1982). 